\title{
A Case of Adenocarcinoma of the Endometrium Extending into the Leiomyoma of the Uterus in a Rabbit
}

\author{
Tetsuro KUROTAKI ${ }^{1) *}$, Hiroko KOKOSHIMA ${ }^{1)}$, Fumiyo KITAMORI ${ }^{2)}$, Takashi KITAMORI ${ }^{2)}$ and \\ Minoru TSUCHITANI ${ }^{1)}$ \\ ${ }^{1)}$ Mitsubishi Chemical Safety Institute Ltd., 14 Sunayama, Kamisu-shi, Ibaraki 314-0255 and ${ }^{2)}$ Kitamori Animal Hospital, 2368 \\ Kobayashi, Mobara-shi, Chiba 297-0074, Japan
}

(Received 21 December 2006/Accepted 21 May 2007) ABSTRACT. In a pet rabbit, 2 tumor masses one on each horn were macroscopically seen in the wall of the uterus. On light microscopic
examination, the right horn mass consisted of an admixture of neoplastic epithelial and mesenchymal element. The epithelial element
was composed of neoplastic epithelial cells with numerous mitotic figures and formed varied sizes of acini, glandular, and solid struc-
tures. The tumor was diagnosed as an adenocarcinoma of the endometrium. The mesenchymal element was composed of well-differen-
tiated smooth muscle cells and was diagnosed as a leiomyoma. While adenocarcinoma cells formed a protrusive mass in the uterine
lumen, they also showed an extension into the leiomyoma of the myometrium. By immunohistochemistry, adenocarcinoma stained pos-
itive for cytokeratin (MNF116) and leiomyoma stained positive for smooth muscle actin, showing a substantial difference in the cyto-
logical nature of these tumor cells. The results may give a further evidence supporting the narrative of the tumor development that an
adenocarcinoma of the endometrium extended into leiomyoma of the uterus. To the author's knowledge, this is the first report describing
this type of combination of two independent tumors in a pet rabbit.

KEY WORDS: adenocarcinoma, leiomyoma, rabbit.

J. Vet. Med. Sci. 69(9): 981-984, 2007

In tumors of the uterus, adenocarcinoma of the endometrium and leiomyoma are the most common spontaneous neoplasm in pet rabbits $[5,9,13]$. Adenocarcinoma (10 cases, $21.3 \%$ ) was the most frequently observed, followed by leiomyoma ( 4 cases, $8.5 \%$ ) of the 47 rabbits with uterine disorders [13]. A report presented a 79\% prevalence of adenocarcinoma among the uterine tumors in rabbits over 5 years of age [3]. However, to the author's knowledge, a mixture of two different kinds of tumors in pet rabbits has not been previously reported. This report describes the pathologic, and immunohistochemical features of an admixture of adenocarcinoma of the endometrium and leiomyoma in the uterus of a pet rabbit.

A 7-year-old female rabbit was referred to the Kitamori animal hospital with bloody vaginal discharge. Clinical examination showed hard palpable abdominal masses. Abdominal radiography demonstrated some masses in the abdominal cavity. As uterine tumors were found during laparotomy, the animal was given an ovariohysterectomy. The uterus with tumor masses and the ovaries were fixed in $10 \%$ formalin, and were submitted to the Pathology Division of Mitsubishi Chemical Safety Institute Ltd., for histopathologic examination. Several transverse sections over the tumor masses and the uterine wall were prepared. Tissues were processed routinely and embedded into paraffin blocks. Four-micrometer-thick sections were cut and stained with hematoxylin and eosin (HE) and reticullin silver impregnation. Immunohistochemical stains were performed

\footnotetext{
* Correspondence to: Kurotaki, T., Mitsubishi Chemical Safety Institute Ltd., 14 Sunayama, Kamisu-shi, Ibaraki 314-0255, Japan.

e-mail:kurotaki@ankaken.co.jp
}

using steam antigen retrieval in citrate buffer $(\mathrm{pH}$ 6.0) and a labeled streptoavidin-biotin system (LSAB2 System-HRP, Dako Japan Ltd., Kyoto, Japan). Primary antibodies were mouse monoclonal antibodies against cytokeratin (predilution; MNF116, DAKO Japan) and smooth muscle actin $(\times$ 100; DAKO Japan), and were incubated at room temperature for $60 \mathrm{~min}$. Diaminobenzidine substrate was used as the detection system. The mitotic index was investigated by the proliferating cell nuclear antigen $(\mathrm{PCNA}, \times 200 ; \mathrm{DAKO}$ Japan) method. Tissue specimens of the uterus from a normal pet rabbit were used as the positive control.

Macroscopically, there were two firm tumors one on each horn in the wall of the uterus. On the cut surface of the right side tumor, $30 \times 15 \times 15 \mathrm{~mm}$ in size, there was a cauliflower-like growth that protruded into the lumen coming into contact with a well-demarcated but non-encapsulated mass in the myometrium (Fig. 1). The left side tumor, $25 \times$ $15 \times 10 \mathrm{~mm}$ in size, was only made up of the cauliflowerlike growth in the endometrium. Histologically, the tumor in the myometrium on the right side appeared a smooth muscle tumor with interlacing bundles of muscle fibers. The tumor cells seemed to be well differentiated with abundant eosinophilic cytoplasm, elongated nuclei, and indistinct cell borders (Fig. 2). There were no mitotic figures, and the mitotic index examined by the PCNA method was low. In the silver staining, argyrophil fibers separated individual cells from each other. The fibers stained positive for smooth muscle actin. The tumor was diagnosed as a leiomyoma. The histology of the tumor that protruded into the uterine lumen was characterized by the features of tubular adenocarcinoma with moderate atypia and elaborated by a small amount of fibrous or myxoid connective tissue stroma (Fig. 3). These 

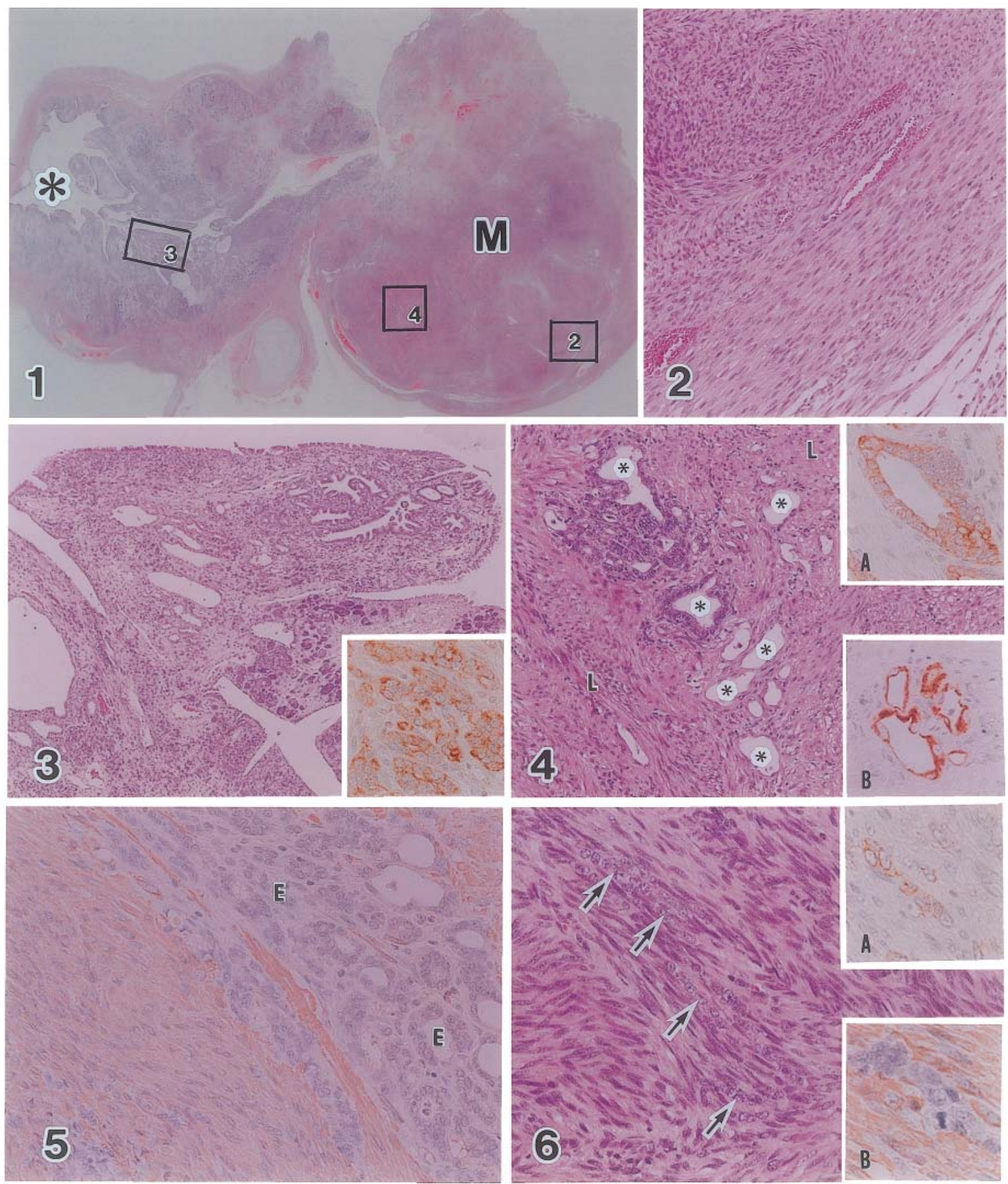

tumor cells stained positive for cytokeratin (Fig. 3, inset) and the mitotic index examined by the PCNA method was high. The tumor developed extensively in the endometrium and extended deeply into the myometrium, and was diagnosed as an adenocarcinoma of the endometrium. In addition, clusters of neoplastic epithelial cells with moderate atypia were noted within leiomyoma. These clusters varied greatly in size and structures and were supported by the well developed stroma (Fig. 4). The glandular structures were lined by a cuboidal to columnar neoplastic epithelial cells that were frequently multilayered. In some areas there were occasional small cysts with flattened neoplastic epithelial cells in the clusters. These cells stained strongly positive for cytokeratin (Fig. 4, insets A, B) and the mitotic index exam- 
ined by the PCNA method was high, but negative for smooth muscle actin (Fig. 5). The other clusters provided solid and small nest structures and lost the epithelial lining nature (Fig. 6). These cells stained weakly positive to negative for cytokeratin (Fig. 6, inset A), negative for smooth muscle actin (Fig. 6, inset B), and positive for PCNA. No transition of the neoplastic epithelial cells to leiomyoma cells was observed. Histological and immunohistochemical characteristics of the neoplastic epithelial cells in the leiomyoma were very similar to those of the adenocarcinoma. As there were, moreover, frequent areas of positional connection between these two proliferations, it was quite plausible that these two neoplastic epithelial tissues should be originated from the same tumor mass, uterine adenocarcinoma, arising from endometrium. The tumor of the left horn was diagnosed as an adenocarcinoma same as that of the right horn. A large number of minuscule foci of adenocarcinoma were also recognized microscopically in the left horn. Therefore, the adenocarcinoma of the endometrium was a multiple tumor.

The topical tumor was composed of epithelial and mesenchymal elements and the former infiltrated into the latter. The histological features of this tumor may suggest Mullerian tumors in rabbits [4] as well as other animals [6, 10, 14]. Mullerian tumors are characterized by an admixture of epithelial and mesenchymal elements. Mixed Mullerian tumors are categorized as adenofibroma, adenosarcoma, carcinofibroma, or carcinosarcoma, depending on whether epithelial and mesenchymal components are benign or malignant [1]. This case may be misdiagnosed as a carcinofibroma due to an admixture of adenocarcinoma and benign mesenchymal tumor, although the latter was not a fibroma but a leiomyoma. However, it was shown that the adenocarcinoma of the endometrium and epithelial tumor in the leiomyoma were similar in morphology, probably originating from the same adenocarcinoma in the endometrium. Though the stromal cells increased slightly, they did not actively proliferate. The leiomyoma cells were well-differentiated and were not relevant to the stromal cells. Therefore, the present case was not diagnosed as a Mullerian tumor.

Collision tumors have been described in various tissues [8]. They are rare in the female genital tract and usually documented as individual case reports. Collision is generally used for the tumor where two kinds of malignant tumors collided. It doesn't seem that the definition of the collision tumor agrees with the tumor colliding between malignant and benign tumors. In the uterus, a benign mixed mesenchymal tumor (angiomyolipoma) that occurred simultaneously with a malignant mullerian tumor has been reported. This tumor was composed of two distinct and separated parts and was considered to be a collision of two neoplasms rather than a malignant transformation of the benign mixed mesenchymal tumor [15]. A collision tumor consisting of three neoplasms at the esophagogastric junction has been reported [11]. Although the majority of the squamous carcinoma was in the esophagus and that of the adenocarcinoma was in the stomach, a broad collision area was seen. Besides, a leiomyoma was noted within the squamous carcinoma. This case had three histologically distinct tumors but no transition was observed between any of them. The tumor was diagnosed as a collision tumor with three components. For the same viewpoint, our case should also be classified as a collision tumor.

There is no known predisposing factor for either tumor. In the uterine adenocarcinoma, the incidence increases with age and nearly all breeds can be affected. The carcinogenic effects of estrogens have been implicated in the evolution of uterine cancer [12]. In another study, however, the administration of estrogens to female Dutch rabbits actually reduced the incidence of endometrial adenocarcinomas [2, 12]. The role of estrogens in development of this tumor is equivocal. The leiomyoma is frequently associated with ovarian follicular cyst or estrogen-secreting tumors [7]. However, there is as yet no precise knowledge of the role of estrogen in tumorigenesis of the uterus.

\section{REFERENCES}

1. Anderson, M. C. 2002. pp. 361-387. In: Pathology of the Female Reproductive Tract (Robboy, S. J., Anderson, M. C. and Russell, P. eds.). Churchill Livingstone, London, UK.

2. Baba, N. and von Haam, E. 1972. Am. J. Pathol. 68: 653-656.

3. Elsinghorst, T. A., Timmermans, H. J. and Hendriks, H. G. 1984. Vet. Q. 6: 200-208.

4. Goto, M., Nomura, Y., Une, Y. and Goldschmidt, M. H. 2006. Vet. Pathol. 43: 560-564.

5. Jones, T. C., Hunt, R. D. and King, N. W. 1997. pp. 11701172. In: Veterinary Pathology, 6th ed. (Jones, T. C., Hunt, R.

Fig. 1. Low magnification of the uterine right side tumor. There is a cauliflower-like growth protruding into the lumen $\left({ }^{*}\right)$ and coming into contact with a well-demarcated but non-encapsulated mass $(\mathrm{M})$ in the myometrium. HE stain. $\times 4.4$.

Fig. 2. Higher magnification of boxed area 2 in Fig. 1. The tumor in the myometrium is diagnosed as a leiomyoma. The tumor cells are well differentiated, have abundant eosinophilic cytoplasm, elongate nuclei, and indistinct cell borders. HE stain. $\times 100$.

Fig. 3. Higher magnification of boxed area 3 in Fig. 1. The histological appearance of the tumor protruding into the uterine lumen. HE stain. $\times$ 40. Inset: This tumor cell stained positive for cytokeratin (MNF116). Immunostaining for cytokeratin (MNF116). $\times 200$.

Fig. 4. Higher magnification of boxed area 4 in Fig. 1. Clusters of neoplastic epithelial cells (*) are noted within leiomyoma (L) with moderate atypia. HE stain. $\times 100$. Insets: Glandular (inset A) and flattening (inset B) neoplastic epithelial cells stained strongly positive for cytokeratin (MNF116). Immunostaining for cytokeratin (MNF116). $\times 200$.

Fig. 5. Cuboidal to columnar neoplastic epithelial cells (E) stained negative for smooth muscle actin. Immunostaining for smooth muscle actin. $\times 200$.

Fig. 6. Solid and small nest structures scattering in the leiomyoma. These lost the epithelial lining nature (arrows). HE stain. $\times 200$. Inset A: Neoplastic epithelial cells stained weakly positive to negative for cytokeratin (MNF116). Immunostaining for cytokeratin (MNF116). $\times$ 200. Inset B: Neoplastic epithelial cells stained negative for smooth muscle actin. Immunostaining for smooth muscle actin. $\times 400$. 
D. and King, N. W. eds.). Williams \& Wilkins, Baltimore.

6. Kaspareit-Rittinghausen, J. and Deerberg, F. 1990. Toxicol. Pathol. 18: 417-422.

7. Kennedy, P. C. and Miller, R. B. 1993. pp. 450-455. In: Pathology of Domestic Animals, vol.3, 4th ed. (Jubb, K. V. F., Kennedy, P. C. and Parmer, N.eds.), Academic Press, New York.

8. Lam, K. Y., Khoo, U-S. and Cheung, A. 1999. Int. J. Gynecol. Pathol. 18: 77-81.

9. MacLachlan, N. J. and Kennedy, P. C. 2002. pp. 558-560. In: Tumors in Domestic Animals, 4th ed. (Meuton, D. J. ed). Iowa State Press, Ames.

10. Nicotina, P. A., Zanghi, A. and Catone, G. 2002. Vet. Pathol.
39: $158-160$.

11. Pai, S. A., Kher, N. and Krishnamurthy, S. 1997. Indian J. Gastroenterol. 16: 116.

12. Percy, D. H. and Barthold, S. W. 2001. pp. 303-306. In: Pathology of Laboratory Rodents and Rabbits, 2nd ed. Iowa State Press, Ames.

13. Saito, K., Nakanishi, M. and Hasegawa A. 2002. J. Vet. Med. Sci. 64: 495-497.

14. Satoh, H., Kai, K., Yabe, K., Fujii, F. and Furuhama, K. 2003. Toxicol. Pathol. 31: 179-184.

15. Waxman, M., Boyce, J. G., Macasaet, M. M. and Lu, T. 1982. Am. J. Clin. Pathol. 77: 631-637. 